\title{
Images
}

\section{A teaspoon in the stomach: How do we proceed from here?}

\author{
Itai Shavit, MD; Danny Eytan, MD; Ron Shaoul, MD
}

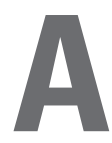

previously healthy 15 -year-old girl presented to the emergency department (ED) 1 hour after ingesting a teaspoon. The spoon was accidentally ingested during sudden forceful inspiration while watching a scary movie at home. The patient was asymptomatic and her physical examination was unremarkable. A plain abdominal radiograph was obtained (Fig. 1).

Two options were considered for extraction: endoscopy and gastrostomy. We decided to attempt an endoscopic removal at the ED. The procedure was performed by a skilled gastroenterologist with the patient deeply sedated by an experienced emergency physician. The spoon, which was covered with food particles, was identified and grasped with a polypectomy snare at the side of the handle (Fig. 2) and was slowly retrieved through the lower esophageal sphincter. When it reached the hypopharynx level, the teaspoon was manually grasped by the emergency physician, who carefully pulled it out while maintaining the open airway.

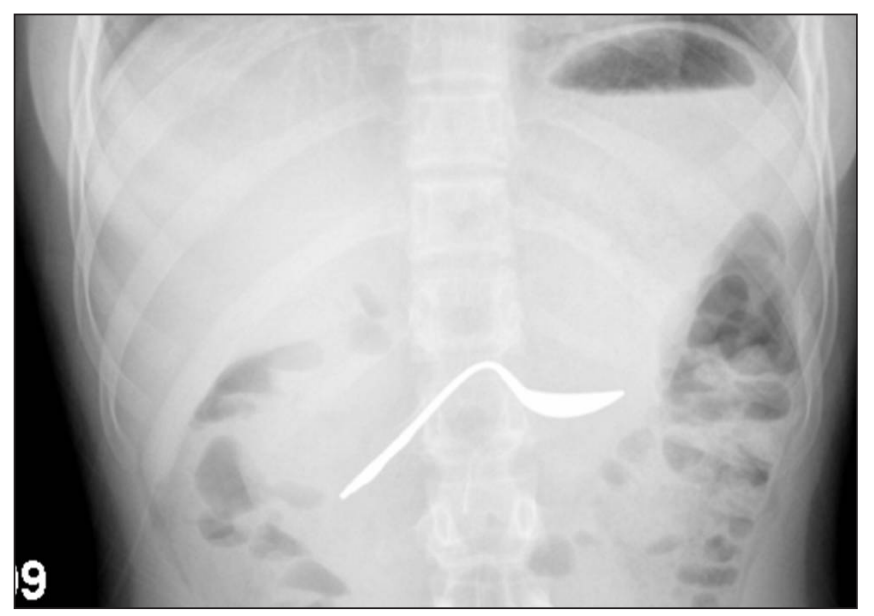

Fig. 1. Plain radiograph of the abdomen of a 15-year-old girl showing a bent metal spoon in the stomach.
Unlike most cases of foreign-body ingestion, there have been no cases reported of spontaneous passage of a teaspoon..$^{1-5}$ Because of problems related to orientation and grasping, there have been only a few reports of endoscopic removal of long foreign bodies such as teaspoons..$^{2-4}$ A retrospective analysis of 542 cases of foreign-body ingestion recommended surgical removal in cases in which the spoon handle is longer that $6 \mathrm{~cm}{ }^{1}$ Although this patient underwent a successful endoscopic removal at the ED, gastrostomy should always be considered when a long object, such as a teaspoon, is lodged in the stomach.

Competing interests: None declared.

Keywords: ingestion, foreign body, endoscopy, removal

\section{REFERENCES}

1. Velitchkov NG, Grigorov GI, Losanoff JE, et al. Ingested foreign bodies of the gastrointestinal tract: retrospective analysis of 542 cases. World J Surg 1996;20:1001-5.

2. Aoyagi K, Maeda K, Morita I, et al. Endoscopic removal of a

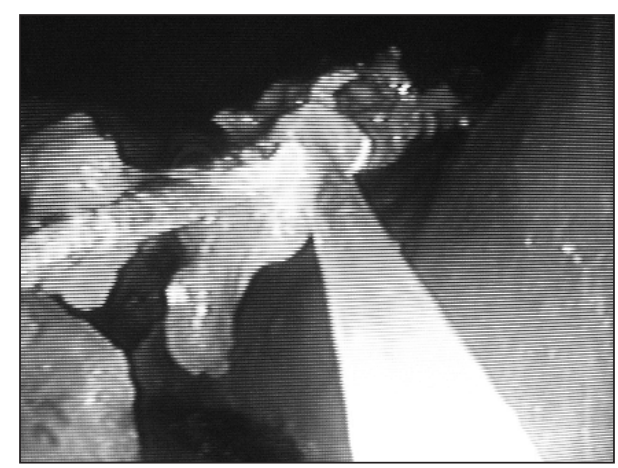

Fig. 2. Endoscopic view of the teaspoon (covered with food particles) grasped by a polypectomy snare at the edge of the handle.

From the Emergency Department, Meyer Children's Hospital, Rambam Medical Center, Haifa, Israel

Submitted Sep. 27, 2009; Accepted Oct. 22, 2009

This article has been peer reviewed.

CJEM 2010;12(5):447-8 
spoon from the stomach with a double-snare and balloon. Gastrointest Endosc 2003;57:990-1.

3. Chang DK, Ross WB. Endoscopic retrieval of a teaspoon from the stomach. ANZ J Surg 2004;74:1013-4.

4. Beldholm BR, Lee AU. Simple endoscopic technique for retrieving a long foreign body from the stomach. ANZ J Surg 2007;77:560-1.
5. Lin CK, Lee KS, Kuo MC, et al. Removal of a misswallowed long spoon via gastrotomy - a case report. Kaobsiung 7 Med Sci 2002;18:208-10.

Correspondence to: Dr. Itai Shavit, P.O. Box 369, Nahariyya 22103, Israel; itai@pem-database.org

\section{RENSEIGNEMENTS AUX LECTEURS}

\begin{abstract}
Abonnement et ventes
Le Journal canadien de la médecine d'urgence (JCMU) est offert à titre gracieux aux membres de l'Association canadienne des médecins d'urgence (ACMU) dont la cotisation est à jour; les autres peuvent s'abonner annuellement. Tarifs pour 2011 (6 numéros) : Abonnements au Canada : individuels 225 \$, établissements 495 \$; aux États-Unis et ailleurs : individuels 350 \$US, établissements 550 \$US. Communiquez avec le bureau de l'ACMU au $800463-$ 1158. Exemplaire unique d'un numéro de l'année en cours 50 \$; anciens numéros $50 \$$ (sujet à disponibilité). On doit faire le paiement à l'ordre de l'ACMU en argent canadien ou américain. Les cartes VISA et MasterCard sont également acceptées.
\end{abstract}

\section{Changement d'adresse}

Nous demandons un avis de 6 à 8 semaines afin d'assurer un service ininterrompu. Veuillez faire parvenir votre adresse postale actuelle, votre nouvelle adresse et la date à laquelle elle doit entrer en vigueur à : cjem@caep.ca ou faites parvenir un fax au 613 523-0190.

\section{Tirés à part}

Des tirés à part d'articles du $7 C M U$ sont disponibles en quantités minimales de 50. Pour des renseignements sur les commandes, veuillez communiquer avec la coordonnatrice des tirés à part, $800663-7336 \mathrm{ou}$ 613 731-8610 x2110, fax 613 565-7704, janis.murrey@cma.ca

\section{Disponibilité électronique}

Le JCMU est disponible sur le site Web de l'ACMU (cjemonline.ca).

\section{Répertoriage}

Le JCMU est répertorié par MEDLINE/PubMed, Embase,
CINAHL, International Pharmaceutical Abstracts, BIOME/ OMNI, Scirus, Cochrane Prehospital and Emergency Health Field et Pubs Hub.com.

\section{Droits d'auteur et permissions}

Le droit d'auteur de tout le matériel appartient à l'ACMU ou à ses concédants. Vous pouvez en général reproduire ou utiliser le matériel trouvé dans ce journal seulement à condition de respecter la loi canadienne sur le droit d'auteur et d'accorder le crédit à l'auteur original. Pour photocopier le document ou le reproduire autrement, veuillez communiquer avec la Canadian Copyright Licensing Agency (Agence canadienne d'octroi des licences pour le droit d'auteur) (Access Copyright) au 800 893-5777, access copyright.ca. Pour toute autre utilisation, y compris la réédition, la redistribution, le stockage dans un système de consultation ou la transmission sous quelque forme ou par quelque moyen que ce soit, veuillez communiquer avec Andrea Schaffeler, Rédactrice administrative, Canadian Journal of Emergency Medicine, 628 Cowan Circle, Pickering ON L1W 3K7; cjem@rogers.com

\section{Instructions pour les auteurs}

Visitez le www.cjem-online.ca.

\section{Advertising}

Annonces classées : Communiquez avec le service de publicité du Journal, JCMU, 1867, prom. Alta Vista, Ottawa ON K1G 5W8; 800 663-7336 ou 613 731-8610; fax 613 565-7488; advertising@cma.ca. Veuillez consulter la section des Annonces classées du Journal pour tout renseignement sur les tarifs. Annonces publicitaires : Communiquez avec le service de publicité du Journal (voir l'information précédente). 